\title{
Clinical outcomes of non-exposed endoscopic wall-inversion surgery for gastric submucosal tumors: long-term follow-up and functional results
}

\author{
Junya Aoyama ${ }^{1}$ - Osamu Goto ${ }^{2,3}$ (1) - Hirofumi Kawakubo ${ }^{1} \cdot$ Shuhei Mayanagi ${ }^{1} \cdot$ Kazumasa Fukuda $^{1} \cdot$ Tomoyuki Irino $^{1}$. \\ Rieko Nakamura ${ }^{1} \cdot$ Norihito Wada $^{1} \cdot$ Hiroya Takeuchi ${ }^{1,4} \cdot$ Naohisa Yahagi $^{2} \cdot$ Yuko Kitagawa $^{1}$
}

Received: 21 March 2019 / Accepted: 28 June 2019 / Published online: 3 July 2019

(c) The International Gastric Cancer Association and The Japanese Gastric Cancer Association 2019

\begin{abstract}
Background and aims To avoid the risk of iatrogenic dissemination during procedures, we have developed a combined laparoscopic and endoscopic surgery with a nonexposure technique for resection of gastric tumors. The study aim was to evaluate the feasibility and safety of non-exposed endoscopic wall-inversion surgery (NEWS) for gastric submucosal tumors (SMTs). Methods Between August 2013 and February 2018, NEWS was performed for 42 patients with gastric SMTs $\leq 3 \mathrm{~cm}$ in diameter at our institution. We retrospectively investigated the patients' backgrounds, operative and perioperative outcomes, tumor pathological characteristics, and follow-up data.

Results All tumors were resected with negative margins by NEWS. The median operation time was 198 min, and the median estimated blood loss was $5.0 \mathrm{~mL}$. Adverse events occurred in one patient with pneumonia. All patients were alive without recurrence within the median follow-up period of 29.2 months. The average body weight loss rate was $0.3 \pm 4.0 \%$. No food residue was observed at endoscopic follow-up.

Conclusions On the basis of slight body weight loss and the absence of food residue observed in the postoperative endoscopy, NEWS appeared to be safe and feasible for gastric SMTs and to preserve function of the remnant stomach.
\end{abstract}

Keywords Gastric submucosal tumors · Laparoscopic endoscopic co-operative surgery $\cdot$ Gastric remnant function

\section{Introduction}

In most gastric submucosal tumors (SMTs), including potentially malignant gastrointestinal stromal tumors (GISTs), the risk of nodal metastasis is considered to be low [1-3].

Osamu Goto

o-goto@nms.ac.jp

1 Department of Surgery, Keio University School of Medicine, 35 Shinanomachi, Shinjuku-ku, Tokyo 160-8582, Japan

2 Division of Research and Department for Minimally Invasive Treatment, Cancer Center, Keio University School of Medicine, 35 Shinanomachi, Shinjuku-ku, Tokyo 160-8582, Japan

3 Department of Gastroenterology, Nippon Medical School, 1-1-5 Sendagi, Bunkyo-ku, Tokyo 113-8603, Japan

4 Department of Surgery, Hamamatsu University School of Medicine, 1-20-1 Handayama, Higashi-ku, Hamamatsu 431-3192, Shizuoka, Japan
Therefore, partial resection without lymphadenectomy is accepted as the standard treatment for patients with gastric SMTs [4].

Endoscopic full-thickness resection (EFTR) with or without laparoscopic assistance is a promising technique used to minimize the gastric resection size by visualizing a demarcation line of the lesion from the inside of the lumen endoscopically [5, 6]. However, particularly in conventional EFTR, it requires intentional perforation during the procedure, which might cause bacterial contamination in the abdominal space and cause tumor cell seeding in ulcerated SMTs or cancers. With the aim of avoiding the risk of iatrogenic dissemination, non-exposed endoscopic wall-inversion surgery (NEWS) has been developed as a new full-thickness resection technique [7-9].

Since the concept was first reported in 2011 [7] and then clinically introduced in Japan [9], awareness of NEWS has been gradually increasing around the world [10-12]. The safety and feasibility of this procedure have been shown over the short term $[13,14]$. However, limited information 
is available for the long-term results of NEWS for gastric SMTs, including prognosis and gastric function. The aim of the study was to retrospectively analyze patients who underwent NEWS for gastric SMTs to investigate the feasibility and safety of this technique with long-term outcomes.

\section{Patients and methods}

\section{Patients}

We retrospectively collected the data of 42 patients with gastric SMTs who underwent NEWS at Keio University Hospital between August 2013 and February 2018. One patient underwent NEWS for two simultaneous lesions, respectively. Therefore, 42 patients and 43 lesions were included in the study. Target lesions were preoperatively evaluated by endoscopy, computed tomography (CT), and endoscopic ultrasound. NEWS was indicated for intraluminal or intramural growth-type gastric SMTs with a maximum diameter of $\leq 3 \mathrm{~cm}$, regardless of the location, that were considered to be surgical indications according to the Japanese GIST guideline [4]. In this guideline, SMTs should be treated when at least one of the following criteria was met: having any symptoms, pathologically diagnosed as having GISTs, growing tendency, and malignant findings by endoscopic or endosonographic findings, e.g., ulcerative change, irregular margins, and heterogenous echo pattern. This study was conducted with the approval of the Institutional Review Board of Keio University.

\section{Surgical procedure}

NEWS has been previously described in detail [14]. The patient was placed in the supine position under general anesthesia. A camera port was inserted at the umbilicus, and four trocars were inserted into the left-upper, left-lower, right-upper, and right-lower quadrants. The tumor location was intraluminally confirmed using a flexible endoscope. After making the mucosal markings endoscopically around the lesion, the serosal markings were made laparoscopically just behind the mucosal markings with endoscopic navigation. Subsequently, hyaluronic acid solution with a small amount of indigo carmine was injected into the submucosal layer circumferentially around the lesion. The seromuscular layer was cut circumferentially along the serosal markings laparoscopically. After the circumferential seromuscular incision was completed, the seromuscular layer was linearly sutured laparoscopically, with the lesion inverted toward the inside of the lumen. Finally, circumferential mucosal and submucosal incisions around the mucosal markings were made endoscopically, and the full-thickness incision was completed. The resected lesion was retrieved perorally. The procedure was finished by closing the mucosal defect with clipping or endoscopic hand suturing [15].

\section{Postoperative management}

The condition of each patient was carefully monitored daily. Blood tests were performed on the day after the surgery and thereafter as necessary. After discharge from the hospital, the patients were generally followed up 1 month later and then every 6 months at the outpatient clinic. Endoscopy was performed every 6 months for all patients, and CT was performed every 12 months for patients pathologically diagnosed to have GIST with intermediate-to-low risk. For the patients with high-risk GIST, adjuvant chemotherapy with imatinib was initiated, and CT was performed every 6 months according to the Japanese GIST guidelines [4].

\section{Outcome measures}

As short-term outcomes, operation time, estimated blood loss, completeness of the procedure, perioperative adverse events, length of hospital stay, postoperative blood test data, and pathological results, including surgical margin, were examined. Overall survival, recurrence-free survival, body weight at the outpatient visit after surgery, and food residue in follow-up endoscopy were also investigated as long-term outcomes.

\section{Results}

This study included 43 lesions in 42 patients. The lesions were located in the upper $(n=28)$, middle $(n=11)$, or lower $(n=4)$ third of the stomach (Table 1). Among them, 10 lesions had a central depression, including ulcer formation. The mean estimated tumor diameter was $24.6 \mathrm{~mm}$.

All lesions were resected with NEWS as scheduled. The median operation time was $198 \mathrm{~min}$, with a median estimated blood loss of $5.0 \mathrm{~mL}$ (Table 2). These results included a patient in which NEWS was performed for each of two lesions, a patient in which NEWS and laparoscopic and endoscopic co-operative surgery (LECS) were performed for each lesion, and a case in which laparoscopic cholecystectomy for cholecystolithiasis was simultaneously performed. There were no severe adverse events during the procedure, although perforation occurred during laparoscopic seromuscular incision in one $(2.3 \%)$ patient. The median time to discharge of the patients was 7.0 days after the surgery. No severe postoperative adverse events occurred except in one patient who experienced pneumonia (Clavien-Dindo Grade II) on postoperative day 2 .

All tumors were excised en bloc with negative surgical margins (Table 3). The resected SMTs were pathologically 
Table 1 Characteristics of the patients who underwent non-exposed endoscopic wall-inversion surgery

\begin{tabular}{ll}
\hline & Value \\
\hline Age (years), mean \pm SD & $59.5 \pm 13.3$ \\
Sex: $n(\%)$ & \\
Male & $23(55 \%)$ \\
Female & $19(45 \%)$ \\
Lesion location: $n(\%)$ & \\
Upper third & $27(63 \%)$ \\
Middle third & $12(28 \%)$ \\
Lower third & $4(9 \%)$ \\
Circumference, $n(\%)$ & \\
Anterior wall & $14(33 \%)$ \\
Posterior wall & $11(26 \%)$ \\
Greater curvature & $12(28 \%)$ \\
Lesser curvature & $6(14 \%)$ \\
Central depression, including ulcer formation, $n(\%)$ & \\
Absent & $33(77 \%)$ \\
Present & $10(23 \%)$ \\
Tumor size (mm), mean \pm SD & $24.6 \pm 8.6$ \\
\hline
\end{tabular}

$S D$ standard deviation

Table 2 Operative characteristics and perioperative outcomes

\begin{tabular}{ll}
\hline & Value \\
\hline Operation time (min), median (IQR) & $198(173,230)$ \\
Intraoperative blood loss (mL), median (IQR) & $5.0(5.0,5.0)$ \\
Completeness of the procedure, $n(\%)$ & $43(100 \%)$ \\
Intraoperative perforation, $n(\%)$ & $1(2.3 \%)$ \\
Postoperative adverse events, $n(\%)$ & $1(2.3 \%)$ \\
Postoperative hospital stay (days), median & $7.0(6.3,8.0)$ \\
$\quad(\mathrm{IQR})$ & \\
Maximal body temperature $\left({ }^{\circ} \mathrm{C}\right)$, median $(\mathrm{IQR})$ & $37.7(37.3,38.0)$ \\
Maximal WBC $\left(\mathrm{mm}{ }^{3}\right)$, median $(\mathrm{IQR})$ & $9050(7250,10,500)$ \\
Maximal CRP $(\mathrm{mg} / \mathrm{dL})$, median $(\mathrm{IQR})$ & $2.98(1.83,4.68)$ \\
\hline
\end{tabular}

$I Q R$ interquartile range, $W B C$ white blood cell count, $C R P$ C-reactive protein

diagnosed as GISTs $(n=24)$, ectopic pancreas $(n=6)$, leiomyoma $(n=5)$, neurinoma $(n=3)$, granuloma $(n=2)$, hemangioma $(n=1)$, gastritis cystica profunda $(n=1)$, and desmoid fibromatosis $(n=1)$. In addition, 24 GISTs were categorized by Fletcher's classification, which included very low $(n=7)$, low $(n=14)$, intermediate $(n=1)$, and high $(n=2)$ risk. Regarding GISTs classified as high risk, one patient had a tumor of $30 \mathrm{~mm}$ in diameter located at the anterior wall of the upper third of the stomach with a central depression, and the patient was followed up for 42 months. Another patient had a tumor of $30 \mathrm{~mm}$ in diameter located at the posterior wall of the upper third of the stomach without
Table 3 Pathological characteristics

\begin{tabular}{lc}
\hline & Value \\
\hline En bloc resection, $n(\%)$ & $43(100 \%)$ \\
Surgical margin, $n(\%)$ & \\
Positive & $0(0 \%)$ \\
Negative & $43(100 \%)$ \\
Pathological diagnosis, n (\%) & \\
GIST, total & $24(56 \%)$ \\
Very low grade & $7(29 \%)$ \\
Low grade & $14(58 \%)$ \\
Intermediate grade & $1(4 \%)$ \\
High grade & $2(8 \%)$ \\
Ectopic pancreas & $6(14 \%)$ \\
Leiomyoma & $5(12 \%)$ \\
Neurinoma & $3(7 \%)$ \\
Granuloma & $2(5 \%)$ \\
Hemangioma & $1(2 \%)$ \\
Gastritis cystica profunda & $1(2 \%)$ \\
Desmoid fibromatosis & $1(2 \%)$ \\
\hline
\end{tabular}

GIST gastrointestinal stromal tumor

a central depression, and the patient was followed up for 27 months. Both these patients are alive without any recurrence. Among 10 tumors with a central depression, five patients had GISTs, including one at high, one at intermediate, two at low, and one at very low risk. The remaining five tumors were ectopic pancreas, neurinoma, granuloma, hemangioma, and gastritis cystica profunda. Of these, ulcer formation on the top of the tumor surface was found in one GIST with intermediate risk and one neurinoma.

Except for one patient with granuloma, 41 (97.6\%) were followed up for $>6$ months. There was no apparent loss in the quality of life (QOL), and no obvious postoperative stomach syndrome was observed at least in the medical record review in all patients. No recurrence or deaths were reported within the median follow-up period of 29.2 months (Table 4). Totally 39 patients underwent follow-up endoscopy in our hospital after surgery, and no local recurrence was reported in the latest endoscopy at the median follow-up period of 26.3 months after surgery in all patients. In addition, no food residue was reported in the latest endoscopy.

During the median observatory period of 29.2 months, the preoperative and latest median hemoglobin levels were $13.5 \mathrm{~g} / \mathrm{dL}$ and $14.0 \mathrm{~g} / \mathrm{dL}$, respectively, without a significant difference $(p=0.964)$. In addition, during the same observatory period, the preoperative and latest median albumin levels had no significant difference $(4.4 \mathrm{~g} / \mathrm{dL}$ and $4.2 \mathrm{~g} / \mathrm{dL}$, respectively, $p=0.162$ ).

The change in body weight at 6 months and latest outpatient visit were reviewed. At 6 months (median observational period, 7.1 months), the weights of 33 (78.5\%) 
Table 4 Long-term outcomes after the surgery

\begin{tabular}{ll}
\hline & Value \\
\hline Overall survival & $100 \%$ \\
Recurrence & $0 \%$ \\
Observation period (months), median (IQR) & $29.2(24.5,40.9)$ \\
Body weight loss $>10 \%, n$ & $0 / 38$ \\
Body weight decrease, mean $\pm \mathrm{SD}$ & $-0.3 \pm 4.0 \%$ \\
Observation period (months), median (IQR) & $7.1(6.5,8.7)$ \\
Body weight loss $>10 \%, n$ & $2 / 33$ \\
Body weight decrease, mean $\pm \mathrm{SD}$ & $-1.7 \pm 3.9 \%$ \\
Food residue in follow-up endoscopy, $n$ & $0 / 39$ \\
Preoperative hemoglobin (g/dL), median (IQR) & $13.5(12.9,14.9)$ \\
Hemoglobin in follow-up blood test (g/dL), median (IQR) & $14.0(13.0,14.7)$ \\
Preoperative albumin (g/dL), median (IQR) & $4.4(4.2,14.5)$ \\
Albumin in follow-up blood test (g/dL), median (IQR) & $4.2(4.1,4.4)$ \\
\hline
\end{tabular}

$I Q R$ interquartile range, $S D$ standard deviation patients were collected from the medical records. The average weight loss was $1.7 \%$, and two $(4.8 \%)$ patients had a weight loss of $>10 \%$ of the primary weight. For the latest body weight (median observational period, 20.9 months), $38(90.5 \%)$ patients were available. The average weight loss rate was $0.3 \%$, and no patient had a weight loss of $>10 \%$ of the preoperative weight.

\section{Discussion}

The study results demonstrated that NEWS was both oncologically and technically safe, with a small amount of intraoperative blood loss, minimal perioperative complications, mild postoperative inflammation findings, tumor-free margins in all cases, and no recurrence. NEWS was clinically durable with an acceptable operation time and postoperative hospitalization period. In addition, severe weight loss was not observed because of minimal resection and less deformation, which suggests the advantage of NEWS as a functionpreserving surgery. This study includes the largest number of case series on NEWS till date. To the best of our knowledge, this is the first report to provide the long-term follow-up data on NEWS for gastric SMT.

Numerous studies have shown that laparoscopic surgery for gastric SMTs was safe and feasible [16-18]. The surgical outcomes in these reports showed mean operation times of 48.5-169 min, mean blood loss of 100-106 mL, mean length of hospital stay of 3.8-9.1 days, and postoperative complication rates of 2.7-9.5\% [16-18]. Compared with the results of purely laparoscopic gastric resection, the operation time was longer, the complication rate and hospital stay were equivalent, and the estimated blood loss was much smaller for NEWS. Despite the disadvantages of procedural time, NEWS would have the reasonable advantage that tumors can be completely excised with minimal margin by visualizing a demarcation line of the lesion from the inside of the lumen endoscopically. Our results showed that en bloc resection and a negative surgical margin were achieved in all cases and there was no local recurrence, whereas resection with an appropriate line may be difficult with a purely laparoscopic procedure. Indeed, few cases of local recurrence due to incomplete resection have also been reported $[16,18]$. Nishida et al. have reported that recurrence risk after surgery is the highest during the initial few years after surgery, and thereafter the risk gradually decreases [16]; therefore, this series was worthy although the median observational period seemed to be relatively short (29.2 months).

Matsuda et al. conducted a multicenter study in Japan, which revealed safety and feasibility in a conventionally open technique of LECS for gastric SMTs [19]. They reported a mean operation time of 190.2 min, mean blood loss of $15.1 \mathrm{~mL}$, mean length of hospital stay of 9.8 days, a postoperative complication rate of $4.8 \%$, and lack of recurrence [19]. In the present study, we showed nearly equivalent results with NEWS to those of the preceding report. However, depending on the location of the tumor, the working area will be smaller; thus, increasing the difficulty of this technique. For example, the tumors located at the posterior wall or just below the cardia are difficult to be operated on mainly because of the laparoscopic aspects, whereas the tumors located in the lower third of the stomach are difficult to be accessed by the endoscopists. In addition, the seromuscular closure should be along the longitudinal direction of the stomach to avoid gastric deformity as much as possible.

LECS can be applied to a tumor with a diameter of $\geq 5 \mathrm{~cm}$, whereas NEWS is limited by the size of the tumor and applied to tumors with diameters of $\leq 3 \mathrm{~cm}$ because of transoral retrieval of the resected specimen. On the other hand, NEWS has an advantage of being a safer procedure 
from the aspect of nonexposure technique. In other words, NEWS can avoid bacterial contamination in the abdominal cavity and seeding tumor cells to it in ulcerated SMTs or cancers, whereas conventional LECS may theoretically cause it because of intentional perforation during the procedure. For abdominal contamination, Shoji et al. reported that postoperative serum C-reactive protein level was significantly lower, postoperative hospitalization significantly shorter, and operation costs significantly lower in the NEWS group compared with the LECS group [20]. Regarding tumor cell seeding, Hirao et al. reported two patients with early gastric cancer who had recurrence of peritoneal dissemination that seemed to be due to perforation during endoscopic submucosal dissection [21]. In addition, Goto et al. showed stamp cytology results suggesting that cancer cells, including cancer stem cells, could be easily detached by contact with the tumor surface [22]. Although a case series reported that there was no peritoneal recurrence in 90 cases of intraoperative perforation during endoscopic resection for early gastric cancer [23], intentional perforation should be avoided in EFTR especially for ulcerated SMTs or gastric cancers. Indeed, our results showed that GIST with ulceration included intermediate case in Fletcher's classification. Therefore, given the principle of surgical resection of GIST with an intact pseudocapsule, these tumors should be excised using the nonexposure technique. We revealed that NEWS can achieve surgical results comparable to those of conventional LECS without concern about intraperitoneal bacterial contamination and dissemination of tumor cells.

In this study, our long-term follow-up results demonstrated that NEWS provided a good functional prognosis. Compared with the accumulation of surgical and oncological evidence, little is known about functional outcome for resection of gastric SMTs. With regard to change in body weight, previous reports have shown that weight loss was $5.4 \%$ in the laparoscopic-assisted distal gastrectomy group [24] and 6.9\% in the pylorus-preserving gastrectomy group [25]. Hirota et al. reported severe body weight loss after laparoscopic partial gastrectomy for gastric SMTs was observed in 3 of the 49 patients, and the average weight loss rate was 1.8-2.2\% [26]. Our results showed no significant body weight loss after NEWS, and the average weight loss rate was $0.3 \%$; that is, the body weight had scarcely decreased even after NEWS in most patients. This finding suggested that there was almost no influence of NEWS on gastric function because the gastric volume was maintained and the stomach was minimally deformed. No food residue was observed in all 39 patients who underwent follow-up endoscopy, which also demonstrated good function of the remnant stomach.

Surgical excision for small-sized GIST is still controversial. Furthermore, nonexposure technique may not be attached to SMTs even with ulceration. However, once iatrogenic abdominal contamination or peritoneal seeding occurs, the prognosis may become worse or at least it would take longer time for the condition to improve than the procedural time additionally required in this technique. Considering these possible risks, the disadvantage of the operation time is acceptable if the procedure can sufficiently maintain the postoperative QOL without the risk of peritoneal contamination or dissemination.

There were several limitations in this study. First, the study was a retrospective case series conducted at a single institution. Second, the procedure was performed by skilled laparoscopists and endoscopists, and the required laparoscopic/endoscopic skill level to successfully perform NEWS is unknown. Third, this was a single-arm study without comparison with other conventional surgical techniques. Fourth, it would be better to directly evaluate the usefulness of this minimally invasive surgery using a QOL evaluation chart. Further prospective studies, including QOL assessment, are desirable to demonstrate the advantages of NEWS, particularly with respect to the patient's QOL.

In conclusion, our study results showed that, on the basis of the acceptable operation time, extremely low blood loss, minimal adverse events, and lack of recurrence as well as slight body weight loss and absence of food residue, NEWS was feasible, safe, and useful for curative resection of gastric SMTs and preservation of gastric function. NEWS appears to be a promising less-invasive, function-preserving, local resection technique for use even in ulcerated SMTs or cancers.

\section{Compliance with ethical standards}

Conflict of interest The authors report no conflicts of interest in this work.

\section{References}

1. Joensuu H, Fletcher C, Dimitrijevic S, Silberman S, Roberts P, Demetri G. Management of malignant gastrointestinal stromal tumours. Lancet Oncol. 2002;3:655-64.

2. Iii CEW, Brock GN, Fan J, Byam JA, Scoggins CR, McMasters KM, et al. An evaluation of 2537 gastrointestinal stromal tumors for a proposed clinical staging system. Arch Surg. 2009; $144: 670-8$.

3. DeMatteo RP, Lewis JJ, Leung D, Mudan SS, Woodruff JM, Brennan MF. Two hundred gastrointestinal stromal tumors recurrence patterns and prognostic factors for survival. Ann Surg. 2000;231:51-8.

4. Nishida T, Hirota S, Yanagisawa A, Sugino Y, Minami M, Yamamura $\mathrm{Y}$, et al. Clinical practice guidelines for gastrointestinal stromal tumor (GIST) in Japan: English version. Int J Clin Oncol. 2008:13:416-30.

5. Schlag C, Wilhelm D, von Delius S, Feussner H, Meining A. EndoResect study: endoscopic full-thickness resection of gastric subepithelial tumors. Endoscopy. 2012;45:4-11. 
6. Hiki N, Yamamoto Y, Fukunaga T, Yamaguchi T, Nunobe S, Tokunaga $\mathrm{M}$, et al. Laparoscopic and endoscopic cooperative surgery for gastrointestinal stromal tumor dissection. Surg Endosc. 2008;22:1729-35.

7. Goto O, Mitsui T, Fujishiro M, Wada I, Shimizu N, Seto Y, et al. New method of endoscopic full-thickness resection: a pilot study of non-exposed endoscopic wall-inversion surgery in an ex vivo porcine model. Gastr Cancer. 2011;14:183-7.

8. Mitsui T, Goto O, Shimizu N, Hatao F, Wada I, Niimi K, et al. Novel technique for full-thickness resection of gastric malignancy: feasibility of nonexposed endoscopic wall-inversion surgery (NEWS) in porcine models. Surg Laparosc Endosc Percutan Tech. 2013;23:e217-e221221.

9. Mitsui T, Niimi K, Yamashita H, Goto O, Aikou S, Hatao F, et al. Non-exposed endoscopic wall-inversion surgery as a novel partial gastrectomy technique. Gastric Cancer. 2013;17:594-9.

10. Kim DW, Kim JS, Kim BW, Jung JY, Kim GJ, Kim JJ. Nonexposed endoscopic wall-inversion surgery for gastrointestinal stromal tumor of the stomach: first case report in Korea. Clin Endosc. 2016;49:475-8.

11. Mahawongkajit $\mathrm{P}$, Techagumpuch A, Suthiwartnarueput W. Non-exposed endoscopic wall-inversion surgery for a gastrointestinal stromal tumor of the stomach: a case report. Oncol Lett. 2017;14:4746-50.

12. Hajer J, Havlůj L, Whitley A, Gürlich R. Non-exposure endoscopic-laparoscopic cooperative surgery for stomach tumors: first experience from the Czech Republic. Clin Endosc. 2018;51:167-73.

13. Mitsui T, Yamashita H, Aikou S, Niimi K, Fujishiro M, Seto Y. Non-exposed endoscopic wall-inversion surgery for gastrointestinal stromal tumor. Transl Gastroenterol Hepatol. 2018;3:17.

14. Goto O, Takeuchi H, Sasaki M, Kawakubo H, Akimoto T, Fujimoto A, et al. Laparoscopy-assisted endoscopic full-thickness resection of gastric subepithelial tumors using a nonexposure technique. Endoscopy. 2016;48:1010-5.

15. Goto O, Sasaki M, Akimoto T, Ochiai Y, Kiguchi Y, Mitsunaga $\mathrm{Y}$, et al. Endoscopic hand-suturing for defect closure after gastric endoscopic submucosal dissection: a pilot study in animals and in humans. Endoscopy. 2017;49:792-7.

16. Matthews BD, Walsh RM, Kercher KW, Sing RF, Pratt BL, Answini GA, et al. Laparoscopic vs open resection of gastric stromal tumors. Surg Endosc. 2002;16:803-7.

17. De Vogelaere K, Hoorens A, Haentjens P, Delvaux G. Laparoscopic versus open resection of gastrointestinal stromal tumors of the stomach. Surg Endosc. 2013;27:1546-54.
18. Hu J, Or BH, Hu K, Wang ML. Comparison of the post-operative outcomes and survival of laparoscopic versus open resections for gastric gastrointestinal stromal tumors: a multi-center prospective cohort study. Int J Surg. 2016;33(Pt A):65-71.

19. Matsuda T, Nunobe $S$, Kosuga T, Kawahira H, Inaki N, Kitashiro $\mathrm{S}$, et al. Laparoscopic and luminal endoscopic cooperative surgery can be a standard treatment for submucosal tumors of the stomach: a retrospective multicenter study. Endoscopy. 2017;49:476-83.

20. Shoji Y, Takeuchi H, Goto O, Tokizawa K, Nakamura R, Takahashi $\mathrm{T}$, et al. Optimal minimally invasive surgical procedure for gastric submucosal tumors. Gastr Cancer. 2018;21:508-15.

21. Hirao M, Yamada T, Michida T, Nishikawa K, Hamakawa T, Mita E, et al. Peritoneal seeding after gastric perforation during endoscopic submucosal dissection for gastric cancer. Dig Surg. 2018:35:457-60.

22. Goto O, Shimoda M, Sasaki M, Kiguchi Y, Mitsunaga Y, Akimoto $\mathrm{T}$, et al. Potential for peritoneal cancer cell seeding in endoscopic full-thickness resection for early gastric cancer. Gastrointest Endosc. 2018;87:450-6.

23. Ikehara H, Gotoda T, Ono H, Oda I, Saito D. Gastric perforation during endoscopic resection for gastric carcinoma and the risk of peritoneal dissemination. Br J Surg. 2007;94:992-5.

24. Aoyama T, Sato T, Hayashi T, Yamada T, Cho H, Ogata T, et al. Does a laparoscopic approach attenuate the body weight loss and lean body mass loss observed in open distal gastrectomy for gastric cancer? a single-institution exploratory analysis of the JCOG 0912 phase III trial. Gastr Cancer. 2018;21:345-52.

25. Fujita J, Takahashi M, Urushihara T, Tanabe K, Kodera Y, Yumiba T, et al. Assessment of postoperative quality of life following pylorus-preserving gastrectomy and Billroth-I distal gastrectomy in gastric cancer patients: results of the nationwide postgastrectomy syndrome assessment study. Gastric Cancer. 2016;19:302-11.

26. Hirota M, Nakajima K, Miyazaki Y, Takahashi T, Kurokawa Y, Yamasaki M, et al. Clinical outcomes of laparoscopic partial gastrectomy for gastric submucosal tumors. Asian J Endosc Surg. 2015;8:24-8.

Publisher's Note Springer Nature remains neutral with regard to jurisdictional claims in published maps and institutional affiliations. 